\title{
The Role of Surgery in American Burkitt's Lymphoma in Children
}

\author{
By Mark C. Stovroff, Arnold G. Coran, and Raymond J. Hutchinson \\ Ann Arbor, Michigan
}

\begin{abstract}
- The records of 28 children with the pathological diagnosis of American Burkitt's Iymphoma were reviewed. Twentythree of these children ( $82 \%)$ presented with primary abdominal tumors and 5 with disease located in the head and neck. Twelve required an emergency operation for either intestinal obstruction (3), intussusception (5), or appendicitis (4); the others underwent an elective exploration for tissue diagnosis. Ten patients had disease localized to one particular site. Seven of these 10 children underwent complete resection of the tumor including a right colectomy (4), small bowel segmental resection (1), tonsillectomy (1), and appendectomy (1). Eight children had a subtotal resection of the tumor ( $<90 \%$ of tumor burden) and the rest underwent incisional biopsies. Following the diagnosis, all patients received chemotherapy; $8(29 \%)$ also were treated concurrently with radiation therapy. Nineteen patients $(70 \%)$ remain long-term survivors with a mean survival time of 3.6 years. Eight patients died of either recurrent disease (6) or sepsis secondary to their chemotherapy, with a mean survival time of 6 months. Sixteen patients $(57 \%)$ developed complications during their hospitalization that required surgical consultation or intervention lacute renal failure [9], pleural effusion [2], intestinal obstruction [5], gastric outlet obstruction [1], and wound infection [1]). No subsequent treatment of these complications resulted in mortality or morbidity. The significant positive determinant for survival was the initial absence of either bone marrow or central nervous system involvement $(P<.05)$. In those children who had complete resection of their tumor, survival time was greater than 3.7 years. Despite the relatively small number of patients in this series, these results suggest that surgical intervention for either primary control of the tumor or for management of complications occurring during the medical treatment of this disease may be responsible for the increased survival presently seen in children with American Burkitt's lymphoma.
\end{abstract} Copyright 1991 by W.B. Saunders Company

INDEX WORDS: Burkitt's lymphoma.

$\mathbf{T}$ HE ROLE OF SURGERY in the management of American Burkitt's lymphoma remains ill defined. Due to its rapid doubling time, the tumor spreads quickly, thus bringing it to early medical attention. However, Burkitt's lymphoma is extremely sensitive to chemotherapeutic agents that cause an initial rapid tumor dissolution and, often, long-term remission. ${ }^{1}$ In light of this response, chemotherapy has become the primary treatment modality. As a result, the role of surgical resection remains uncertain. Surgery is required to confirm the diagnosis and may be necessary to relieve the common presenting symptoms of an "acute abdomen." In addition, surgery may be needed for removal or debulking of the primary disease, for management of complications of the treatment of the disease, and for the provision of vascular access for nutritional support and/or dialysis.
However, several investigators recently have suggested that surgical intervention with its attendant negative physiological effects and the resultant delay in the initiation of effective chemotherapy may worsen the outcome observed without surgery. ${ }^{2}$ This retrospective analysis attempts to identify and define the role of surgery in the management of American Burkitt's lymphoma.

\section{MATERIALS AND METHODS}

The records of 28 children with the pathological diagnosis of American Burkitt's lymphoma treated at the C.S. Mott Children's Hospital were reviewed. The analysis included age and sex distribution along with a description of initial pathological findings. In addition, the site of the primary lesion and the presence or absence of bone marrow and central nervous system (CNS) involvement wcre identified. The extent of the initial surgery and the extent of disease were characterized in an effort to determine whether or not surgical resection affected outcome. Localized disease was defined as disease confined to one anatomic area. Disseminated disease was defined as disease extending beyond the primary site or metastatic. Surgical procedures were classified as total resection in which all gross tumor was removed, subtotal resection in which $<90 \%$ of all gross tumor was removed, or incisional biopsy. All complications were tabulated.

All patients received chemotherapy, usually including cyclophosphamide, vincristine, and methotrexate with or without corticosteroids. A selected group of patients received external beam radiation therapy in addition. All living patients have been followed-up for at least 2 years and none have been lost to follow-up.

\section{RESULTS}

There were 22 boys and 6 girls with an average age at presentation of 8.4 years (range 2 to 17 years).

Twenty-three $(82 \%)$ of the patients presented with abdominal disease, and the rest with disease confined to the head or neck. Of the 23 patients with primary abdominal disease, 6 had localized tumors and the remaining 17 had diffuse intraabdominal spread. All patients presenting with disease in head or neck had no evidence of dissemination (Table 1).

Twelve patients presented with findings of an acute abdomen requiring emergent operation. This group

From the Section of Pediatric Surgery and the Department of Pediatrics, C.S. Mott Children's Hospital and University of Michigan Medical School, Ann Arbor, MI.

Date accepted: June 19, 1990.

Address reprint requests to Amold G. Coran, MD, Surgeon-inChief, C.S. Mott Children's Hospital, Room F7516, Bax 0245, Ann Arbor, $M I 48109$.

Copyright $\odot 1991$ by W.B. Saunders Company

$0022-3468 / 91 / 2610-0022 \$ 03.00 / 0$ 
Table 1. Site of Primary Tumor

\begin{tabular}{lccc}
\multicolumn{1}{c}{ Site } & $\begin{array}{c}\text { No. of } \\
\text { Patients }\end{array}$ & Localized & Disseminated \\
\hline Abdomen & $23(82 \%)$ & 6 & 17 \\
Head/neck & $5(18 \%)$ & 5 & - \\
Total & $28(100 \%)$ & $11(40 \%)$ & $17(60 \%)$ \\
\hline
\end{tabular}

of 12 included 3 patients with complete intestinal obstruction secondary to tumor matting, 5 with irreducible intussusception with tumor acting as the lead point, and 4 patients with suspected appendicitis. Additional findings in the group of 23 with abdominal disease included an abdominal mass and incomplete small bowel obstructions. One patient presented with an acute onset of hemiparesis secondary to tumor compression of the cord. Four of the 5 patients presenting with tumor in the head or neck were referred for evaluation of a rapidly enlarging neck mass (Table 2).

Five patients required a right colectomy as their original procedure, 4 of whom were cured by the operation. Six patients had either an appendectomy or segmental small bowel resection, of which only 2 had complete resection. In only 1 patient with disease localized to the head and neck was the tumor completely excised. The remaining operations were either subtotal resections or incisional biopsies (Table 3). Of the 23 patients presenting with tumor in the abdomen, 14 remain long-term survivors $(61 \%)$. Six of these 23 had localized disease and underwent complete resection for cure. Five of these 6 remain disease free $(83 \%)$. The 1 nonsurvivor died of sepsis secondary to an intensive chemotherapy regime for initial CNS involvement. Of the 17 patients presenting with disseminated intraabdominal disease, none were able to undergo complete resection of all gross tumor. Six (35\%) underwent subtotal resections and the remaining 11 had only biopsies. Only 53\% of these 17 patients have survived. Furthermore, there was no significant difference in disease-free time between either of these two latter groups $(2.8 v 2.1$ years; Table 4).

\begin{tabular}{lc}
\multicolumn{2}{c}{ Table 2. Mode of Presentation } \\
\hline Acute abdomen & $12(43 \%)$ \\
Intestinal obstruction & 3 \\
Intussusception & 5 \\
Appendicitis & 4 \\
Hemiparesis & $1(4 \%)$ \\
Abdominal mass & $5(18 \%)$ \\
Enlarging neck mass & $4(14 \%)$ \\
Right eye ptosis & $1(4 \%)$ \\
Incomplete intestinal obstruction & $4(14 \%)$ \\
Renal failure & $1(4 \%)$ \\
\hline
\end{tabular}

Table 3. Operations Performed

\begin{tabular}{lccc}
\hline \multicolumn{1}{c}{ Operation } & $\begin{array}{c}\text { No. of } \\
\text { Patients }\end{array}$ & $\begin{array}{c}\text { Complete } \\
\text { Resection }\end{array}$ & $\begin{array}{c}\text { Subtotal } \\
\text { Resection }\end{array}$ \\
\hline Colectomy & 5 & 4 & 1 \\
Appendectomy & 3 & 1 & 2 \\
Small bowel resection & 3 & 1 & 2 \\
Incisional biopsy & 15 & - & - \\
Vascular access & 16 & - & - \\
Dialysis access & 8 & - & - \\
Laminectomy & 1 & - & - \\
Tonsillectomy & 1 & 1 & - \\
Tube thoracostomy & 2 & - & - \\
\hline
\end{tabular}

Among the patients with their primary disease localized to the head and neck, none had initial evidence of dissemination. All patients in this group remain long-term survivors. Interestingly, only one patient who underwent a tonsillectomy had a complete resection. The others underwent biopsy only. None of the treated patients experienced any local recurrence.

All 8 nonsurvivors had intraabdominal disease. Seven had diffuse nonresectable disease at abdominal exploration. In addition, all 8 had early evidence of metatases with involvement of either their bone marrow or central nervous system. Six of these 8 died of recurrent disease despite an initial positive response to chemotherapy. The other 2 deaths were due to sepsis secondary to the immunosuppresive chemotherapy. The median survival time was 6 months following diagnosis.

Sixteen patients required further surgical consultation or intervention during their hospitalization for chemotherapy. Nine children developed some degree of renal failure secondary to tumor lysis. Eight required access surgery for dialysis ( 7 hemodialysis, 1 peritoneal). Two patients with recurrent malignant effusions required tube thoracostomies. Sixteen patients subsequently required long-term vascular access for hyperalimentation and/or chemotherapy. Surgical consultation was obtained in 5 patients who developed signs of intestinal obstruction. Three of these 5 patients were successfully managed conservatively, 1 underwent an enterolysis, and 1 required surgical reduction of an intussusception that could not be reduced hydrostatically. A 6th patient developed biliary obstruction that ultimately required operative diversion (Table 5).

Only one patient developed a postoperative complication after his initial surgical procedure. This was a wound infection following an appendectomy, requiring open drainage and broad-spectrum antibiotics. Furthermore, no subsequent surgical procedure performed during chemotherapy resulted in any morbidity or mortality. 
Table 4. Survival of Patients With American Burkitt's Lymphoma

\begin{tabular}{ccc} 
Site & $\begin{array}{c}\text { No. of } \\
\text { Patients }\end{array}$ & $\begin{array}{c}\text { No Evidence } \\
\text { of Disease (\%) }\end{array}$ \\
\hline Abdominal disease & 23 & $14(61)$ \\
Localized & 6 & $5(83)$ \\
Total resection & 6 & 5 \\
Subtotal resection & 0 & 0 \\
Biopsy & 0 & 0 \\
Disseminated & 17 & $9(53)$ \\
Total resection & 0 & 0 \\
Subtotal resection & 6 & 3 \\
Biopsy & 11 & 6 \\
Head and neck disease & 5 & $5(100)$ \\
Localized & 5 & $5(100)$ \\
Total resection & 1 & 1 \\
Subtotal resection & - & 4 \\
Biopsy & 4 & - \\
\hline
\end{tabular}

\section{DISCUSSION}

American Burkitt's lymphoma is being recognized more frequently in the pediatric population. Although this entity was initially reported in the surgical literature, current treatment is principally medical. The tumor is extremely sensitive to chemotherapy, with a reported response rate of over $60 \% .{ }^{3}$ Because of this favorable response, the role of surgery in the management of this disease has been limited. However, some investigators have contended that surgery does play an important role in the overall management of this disease. ${ }^{4-6}$ The initial report of Magrath et al of 185 patient's treated in Kampala demonstrated that debulking the tumor improved survival. They concluded that this benefit was only achieved when $>90 \%$ of the tumor was removed. ${ }^{4}$ Kemeny et al, in their review, came to a similar conclusion, reporting a significant survival advantage with complete resection of the primary disease. These authors broadened the role of surgery by suggesting that, even in patients with disseminated disease, the surgeon plays an important role in the secondary management of the disease.'

This report examines the role surgery played in the management of 28 patients with American-Type Burkitt's lymphoma treated at the C.S. Mott Chil-

\begin{tabular}{lc}
$\begin{array}{c}\text { Table 5. Complications Requiring Surgery Following Primary } \\
\text { Treatment of American Burkitt's Lymphoma }\end{array}$ \\
\hline Acute renal failure/tumor lysis syndrome & $\begin{array}{c}\text { No. of } \\
\text { Patients }\end{array}$ \\
\hline Intestinal obstruction & 9 \\
Requiring celiotomy & 5 \\
Managed conservatively & 2 \\
Biliary obstruction (requiring surgical diversion) & 3 \\
Wound infection & 1 \\
Pleural effusion & 1 \\
\hline
\end{tabular}

dren's Hospital. All patients required early surgical intervention for either diagnosis and/or management; $70 \%$ remain long-term survivors. Among patients who underwent complete resection of their abdominal tumor, $83 \%$ remain disease free with a median tumor-frec survival of ncarly 4 years. Among those patients with nonresectable disease in the abdomen treated with either incomplete resection or incisional biopsy, only $53 \%$ remain alive. Inspite of the limitations of a retrospective review and the small number of patients in this series, the data suggest that complete resection, when possible, offers the patient an improved survival. However, this may merely reflect the fact that less disease is more easily completely resected.

Nevertheless, even if complete surgical resection was not possible, all patients did require surgical intervention at some time during their treatment. This involved either emergent celiotomy, early biopsy for diagnosis, or the management of the complications arising during chemotherapy. None of these subsequent surgical interventions resulted in significant morbidity or mortality. Furthermore, this intervention may have played a significant role in the improved survival observed.

Bone marrow or CNS involvement signifies metastatic disease and indicates a poor prognosis. ${ }^{7}$ This observation remained true in the present series. Not one patient found to have disease within the bone marrow or CNS was a long-term survivor. This included a patient who underwent an emergent operation with complete resection of gross disease. In addition, no patients who developed recurrent disease received any benefit from either reresection or salvage chemotherapy.

The patients with primary disease in their head or neck represent an interesting group. All remain free of disease regardless of the extent of resection initially done. This may reflect the absence of disseminated disease at presentation. This improved survival may also be due to the fact that Burkitt's lymphoma in the head and neck may represent a different biological entity, despite its similar histology.

The following conclusions may be drawn. Complete resection results in improved survival. In the case of disseminated or metastatic disease, aggressive debulking of the tumor should be avoided and chemotherapy should be instituted primarily. Surgical resection can be done safely and does not cause significant morbidity or mortality. Finally, even if complete resection is not possible, surgery will still be required for management of the complications occurring during the medical treatment of this disease and may contribute to the improved survival observed. 


\section{REFERENCES}

1. Ziegler JL: Burkitt's lymphoma. N Engl J Med 305:735-745, 1981

2. Kaufman BH, Burgert EO, Banks PM: Abdominal Burkitt's lymphoma: Role of early aggressive surgery. J Pediatr Surg 22:671-674, 1987

3. Ziegler JL: Management of Burkitt's lymphoma: An update. Cancer Treat Rev 6:95-98, 1979

4. Magrath IT, Lwanga S, Carswell W, et al: Surgical reduction of tumor bulk in management of abdominal Burkitt's lymphoma. Br Med J 2:308-312, 1974
5. Kemeny MM, Magrath IT, Brennan MF: The role of surgery in the management of American Burkitt's lymphoma and its treatment. Ann Surg 196:82-86, 1982

6. Janus C, Edwards BK, Sariban E, et al: Surgical resection and limited chemotherapy for abdominal undifferentiated lymphomas. Cancer Treat Rep 68:599-605, 1984

7. Nkrumah FK, Perkins IV, Biggar RJ: Combination chemotherapy in abdominal Burkitt's lymphoma. Cancer 40:1410-1416, 1977 